\title{
Artroplastia total de rodilla en pacientes con secuela de fracturas articulares. Resultados del seguimiento a mediano plazo
}

\author{
Harold Simesen de Bielke," Leonel Pérez Alamino," Santiago Aguer," Germán Garabano," \\ Hernán del Sel," Fernando Lopreite* \\ "Staff Especialista de Cadera y Rodilla, Servicio de Ortopedia y Traumatología, Sanatorio Modelo de San Miguel de Tucumán, \\ Tucumán, Argentina \\ ${ }^{*}$ Servicio de Ortopedia y Traumatología, Hospital Británico de Buenos Aires, Ciudad Autónoma de Buenos Aires, Argentina
}

\begin{abstract}
RESUMEN
Introducción: Las fracturas articulares de rodilla son frecuentes y pueden evolucionar a gonartrosis postraumática. El tratamiento en su estadio final es el reemplazo total de rodilla (RTR). Cuando se indica, el especialista se enfrenta a situaciones que predisponen a resultados menos satisfactorios. El objetivo de este estudio fue evaluar los resultados del RTR en pacientes con secuela de fracturas articulares de rodilla, con un seguimiento mínimo de 4 años. Materiales y Métodos: Estudio retrospectivo observacional, entre mayo de 1999 y enero de 2013. Se evaluaron 25 RTR en 24 pacientes (edad promedio 67.1 años) con secuela de fractura articular de rodilla y un seguimiento promedio de 6.1 años. Tres (12\%) tenían cirugías anteriores al RTR. Ocho se resolvieron en dos tiempos. Se empleó un implante constreñido en 4 pacientes (16\%). Se determinaron el KSS, el KSSf y el rango de movilidad preoperatorios y del último control. Se documentó la supervivencia del implante. Resultados: El KSS se incrementó de 38,5 en el preoperatorio a 82,8 en promedio. El KSSf posoperatorio mejoró de 48 a 85 . El rango de movilidad aumentó de un promedio de $7,5-76^{\circ}$ a $1-102,4^{\circ}$ en el posoperatorio. Cuatro casos fueron reoperados. La supervivencia de la prótesis fue del $92 \%$ a los 6.1 años. Conclusiones: El RTR es adecuado para la gonartrosis postraumática en su estadio final, con buenos resultados a mediano plazo. Si se logran una adecuada alineación posoperatoria y un correcto posicionamiento de los componentes, los resultados son satisfactorios y se asemejan a los del RTR por gonartrosis idiopática.
\end{abstract}

Palabras clave: Reemplazo total de rodilla; fractura; artroplastia.

Nivel de Evidencia: IV (Serie de casos)

Total knee arthroplasty in patients with joint fracture sequela: Mid-term follow-up outcomes

\begin{abstract}
Introduction: Knee joint fractures are common in Orthopedics, and may evolve to post-traumatic Osteoarthritis (PTOA). Total Knee Arthroplasty (TKA) is an effective treatment for the end-stage of this process. When indicated, orthopedic surgeons face many situations that may cause unsatisfactory results and affect prosthesis survival. The purpose of our study was to evaluate TKA outcomes on Osteoarthritis secondary to distal femoral and proximal tibial joint fractures, in cases with a minimum follow-up period of 4 years. Materials and Methods: We carried out an observational, retrospective study on TKA patients treated between May 1999 and January 2013. We evaluated 25 TKAs, performed on 24 patients, with knee fracture sequela, with an average follow-up of 6.1 years and an average age of 67.1 years. Three cases (12\%) had required additional surgeries before TKA. Eight cases (32\%) were 2-stage surgeries. Constrained implants were used in four cases (16\%). The Knee Society Score (KSS), the Functional KSS (KSSf) and the Range of Motion (ROM) were evaluated preoperatively and at the last follow-up. Prosthesis survival evaluation was documented. Results: KSS increased from a preoperative average of 38.5 to a postoperative average of 82.8 . Postoperative KSSf increased on average from 48 to 85 . ROM increased on average from $7.5^{\circ}-76^{\circ}$ to $1^{\circ}-102.4^{\circ}$. Four patients required a second surgical procedure. Prosthesis survival was $92 \%$ at a 6.1 -year average follow-up. Conclusion: TKA is an appropriate treatment for advanced PTOA, with good mid-term outcomes. If adequate postoperative alignment and correct component positioning is achieved, expected outcomes are satisfactory and similar to those with TKA in primary osteoarthritis.
\end{abstract}

Key words: Total knee replacement; fracture; arthroplasty.

Level of Evidence: IV (Case series)

Recibido el 6-8-2019. Aceptado luego de la evaluación el 4-9-2019 • Dr. HAROLD SIMESEN DE BIELKE • haroldsimesen@ hotmail.com (ID

Cómo citar este artículo: Simesen de Bielke H, Pérez Alamino L, Aguer S, Garabano G, del Sel H, Lopreite F. Artroplastia total de rodilla en pacientes con secuela de fracturas articulares. Resultados del seguimiento a mediano plazo. Rev Asoc Argent Ortop Traumatol 2020;85(2):107-118. https://doi.org/10.15417/issn. 1852-7434.2020.85.2.1011 


\section{INTRODUCCIÓN}

Las fracturas de fémur distal y de tibia proximal con compromiso articular pueden desarrollar gonartrosis secundaria o postraumática, independientemente del tratamiento que reciban, con incidencias que pueden alcanzar el 20-46\% según distintos autores. ${ }^{1-4}$ Entre los factores predisponentes, se destacan el daño inicial del cartílago por el trauma, la mala alineación residual y la presencia de implantes en posición subcondral, que pueden lesionar la superficie articular; la edad avanzada y la presencia de enfermedad degenerativa articular o meniscectomía anteriores al trauma. ${ }^{5}$

Una opción adecuada para el tratamiento del estadio final de esta entidad es el reemplazo total de rodilla (RTR). La consolidación viciosa, la seudoartrosis, los defectos óseos, la osteosíntesis previa, la infección latente, la artrofibrosis y el compromiso de tejidos blandos periarticulares constituyen un desafío quirúrgico, aumentan la complejidad del procedimiento y, en muchos casos, obligan a utilizar injerto óseo o implantes de revisión..$^{6-8}$ Las primeras publicaciones sobre los resultados del RTR en pacientes con secuela de fracturas articulares arrojaron valores poco alentadores, con una elevada tasa de complicaciones. ${ }^{2,9}$

El objetivo de este estudio fue evaluar los resultados del RTR en pacientes con secuela de fracturas articulares (femoral distal y tibial proximal) operados en nuestro Servicio, con un seguimiento mínimo de cuatro años. Se hizo hincapié en detallar las dificultades técnicas y las complicaciones ocurridas en los pacientes evaluados.

\section{MATERIALES Y MÉTODOS}

Luego del análisis retrospectivo de la base de datos de nuestro Hospital, se identificaron los pacientes operados de RTR después de una fractura articular de rodilla. Entre mayo de 1999 y enero de 2013, en nuestro Servicio, se realizaron 1019 RTR cementados primarios, 25 (2,45\%) de ellos en 24 pacientes con secuela de fractura articular de rodilla ( 1 caso bilateral simultáneo). Dos pacientes fallecieron por causas no relacionadas con el implante, a los 10 y 12 años del RTR, con buena evolución en el último control, por lo que fueron incluidos en el estudio.

Los criterios de inclusión fueron: pacientes con gonartrosis avanzada, asociada o no a seudoartrosis, sometidos a RTR, con antecedentes de fractura articular de rodilla, ya sea tibial proximal o femoral distal, y un seguimiento mínimo de cuatro años. Se excluyó a los pacientes con antecedentes de enfermedad inflamatoria, como artritis reumatoide, y los que recibían algún tratamiento que pudiese afectar la densidad mineral ósea, por ejemplo, agentes quimioterapéuticos, radioterapia, corticoides en altas dosis o metotrexato.

La serie conformada tuvo un seguimiento promedio de 6.1 años (rango 4.3-16.2). Estaba integrada por siete hombres y 17 mujeres, con una edad promedio de 67.1 años (rango 26-87) en el momento de la artroplastia. Se realizaron 12 RTR derecha y 13 izquierda. El intervalo promedio entre la fractura/osteosíntesis y el RTR fue de 9.4 años, con un amplio rango entre casos (4 meses-32 años). El diagnóstico preoperatorio y el tratamiento previo recibido se detallan en la Tabla 1.

Uno de los pacientes evaluados que había sufrido una fractura articular femoral distal tratada con placa tipo DCS fue sometido al RTR a los cuatro meses de la osteosíntesis debido al fallo precoz del implante; dos casos presentaron seudoartrosis (platillo tibial interno y cóndilo femoral externo); los restantes desarrollaron artrosis postraumática.

Tres (12\%) tenían otras cirugías anteriores al RTR: uno había sido sometido a movilización bajo anestesia con asistencia artroscópica, en nuestro Hospital, debido al desarrollo de rigidez (rango de movilidad [RM] 30-60 ${ }^{\circ}$ ) a los 4.5 meses de la osteosíntesis de la fractura de platillo tibial interno, y llegó a la artroplastia con un RM de 15-110 . Otro caso con una fractura similar había sufrido una infección quirúrgica, por lo que requirió múltiples limpiezas y tratamiento antibiótico, para luego ser operado nuevamente con diagnóstico de seudoartrosis, con colocación de placa bloqueada más aloinjerto de banco y cirugía de cobertura de partes blandas (colgajo de gemelo interno); todos los procedimientos se habían llevado a cabo en otra institución. El tercer individuo, con fractura supraintercondílea, debió ser intervenido otra vez por una seudoartrosis del tercio distal del fémur extrarticular, a los cuatro años de la cirugía primaria (conversión de placa tipo DCS a clavo endomedular retrógrado), en otro Centro.

Solo ocho (32\%) de los 25 casos fueron planificados para resolver en dos tiempos. El primero fue el paciente sometido a una movilización bajo anestesia, momento en el que se le retiró el implante (placa interna), se tomaron muestras de líquido articular, hueso y tejidos blandos periarticulares, con resultados negativos para infección. El otro paciente fue el operado en varias oportunidades con diagnóstico de infección y seudoartrosis, al que se le retiró la osteosíntesis y se le tomaron muestras óseas, con valores negativos. En otros seis casos, también se retiraron los implantes y se tomaron muestras (resultados negativos para infección) en la cirugía anterior a la artroplastia. 
Tabla 1. Tipo de fractura y tratamiento previo recibido

\begin{tabular}{|c|c|c|c|}
\hline Tipo & Paciente & Fractura & Tratamiento \\
\hline & 1 & Platillo tibial interno & Yeso \\
\hline & 2 & Platillo tibial interno & Yeso \\
\hline & 3 & Platillo tibial interno & RAFI \\
\hline & 4 & Platillo tibial externo & Tornillos canulados + aloinjerto \\
\hline & 5 & Platillo tibial externo & Yeso \\
\hline & 6 & Platillo tibial externo & Yeso \\
\hline & 7 & Platillo tibial externo & RAFI \\
\hline & 8 & Platillo tibial externo & RAFI \\
\hline & 9 & Platillo tibial externo & Yeso \\
\hline & 10 & Platillo tibial externo & RAFI \\
\hline \multirow[t]{14}{*}{ Cerrada } & 11 & Ambos platillos & RAFI \\
\hline & 12 & Ambos platillos & RAFI \\
\hline & 13 & Ambos platillos & RAFI \\
\hline & 14 & Ambos platillos & RAFI \\
\hline & 15 & Supraintercondílea & CEM retrógrado + tornillos canulados \\
\hline & 16 & Supraintercondílea & CEM retrógrado + tornillos canulados \\
\hline & 17 & Supraintercondílea & CEM retrógrado + tornillos canulados \\
\hline & 18 & Supraintercondílea & RAFI \\
\hline & 19 & Supraintercondílea & RAFI \\
\hline & 20 & Supraintercondílea & RAFI \\
\hline & 21 & Supraintercondílea & RAFI \\
\hline & 22 & Supraintercondílea & RAFI \\
\hline & 23 & Cóndilo externo & Yeso \\
\hline & 24 & Platillo tibial interno + supraintercondílea & RAFI \\
\hline Abierta & 25 & Platillo tibial interno & Tracción + yeso \\
\hline
\end{tabular}

RAFI = reducción abierta y fijación interna, $\mathrm{CEM}$ = clavo endomedular.

A fin de detectar infección latente, a toda la serie se le realizaron exámenes físico y de laboratorio. La toma de muestra de líquido articular, ósea y de tejidos blandos no se efectuó en forma rutinaria, solo ante la sospecha de infección: 12 pacientes (en 6 se tomaron muestras seriadas de líquido articular mediante punción y, a otros 6 , se les tomaron muestras de líquido, hueso y tejidos blandos, durante el retiro del material de osteosíntesis antes del RTR).

Todos fueron operados en quirófano de flujo laminar, bajo anestesia raquídea hipotensiva. Se administró profilaxis antibiótica con cefalosporina de primera generación, y también para trombosis venosa profunda/tromboembolismo pulmonar, con heparina de bajo peso molecular.

Se realizó una incisión cutánea anterior con artrotomía pararrotuliana interna. Se procuró mantener un puente de piel de 5-7 cm entre el abordaje del RTR y las incisiones previas (en los casos con cirugía/s previa/s). Solo, en algunos pacientes, se utilizaron los abordajes previos. Se efectuó el balance ligamentario antes de los cortes óseos en los 25 casos, dado que todos tenían un deseje en varo o en valgo. 
En referencia a los gestos de ampliación del aparato extensor, a nivel proximal, se efectuó un recorte del recto de tipo snip en 12 casos (48\%), incisión de Coonse-Adams en uno; y a nivel distal, se realizó una osteotomía de la tuberosidad anterior de la tibia en dos rodillas. ${ }^{10}$ Estos últimos tres pacientes utilizaron un inmovilizador durante 45 días después de la cirugía. En dos oportunidades, se liberó el retináculo lateral para mejorar el encarrilado rotuliano.

Se utilizaron implantes cementados con sacrificio del ligamento cruzado posterior (prótesis estabilizada posterior) y se reemplazó la rótula en todos los casos. En 20 pacientes (80\%), se empleó una prótesis estabilizada posterior primaria (8 PFC All-Poly y 12 Sigma, DePuy® J\&J, Warsaw, IN, EE.UU), cuatro (16\%) fueron constreñidas varo-valgo (PFC Sigma TC3, DePuy® J\&J, Warsaw, IN, EE.UU.) y uno (4\%) recibió un reemplazo con estabilización plus (Sigma Plus, DePuy® J\&J. Warsaw, IN, EE.UU.). La elección se basó en el examen físico, las radiografías convencionales y con estrés (solo en casos inestables) y se decidió durante el acto quirúrgico (mediciones con componentes de prueba). Se implantaron tallos femorales o tibiales en nueve rodillas (36\%), con cementado total.

El $48 \%$ de los pacientes (12 rodillas) tenían un defecto óseo en el momento de la artroplastia. En ocho individuos, se rellenó con cemento por tratarse de defectos contenidos y $<5 \mathrm{~mm}$; dos casos requirieron aloinjerto estructurado estabilizado con tornillos; en los dos restantes, se aplicó un suplemento metálico a nivel femoral.

\section{Análisis}

Se examinó la evolución clínica y radiográfica de la muestra a partir de las historias clínicas y el archivo radiográfico de nuestro Centro. Se analizaron los controles de las 3 semanas, 3 meses, 6 meses, al año y cada dos años de la cirugía.

Para el análisis objetivo de la serie se utilizó el Knee Society Score (KSS), y para la valoración funcional, el KSS funcional (KSSf). Además, se midió el RM del último control y se lo comparó con el RM preoperatorio.

Se utilizaron las radiografías anteroposterior y lateral de mejor calidad del último control para evaluar la alineación y la posición de los componentes, el eje anatómico posoperatorio y el aflojamiento de la prótesis, definido por la presencia de una línea radiolúcida completa o hundimiento femoral o tibial $\geq 2 \mathrm{~mm}$. $^{6}$

El análisis de supervivencia contempló la necesidad de cirugía de revisión por cualquier causa. Se realizó una segunda evaluación que incluyó solo la revisión de causa mecánica.

\section{RESULTADOS}

El KSS promedio preoperatorio fue de 38,5 (rango 15-55). En el último control, se había incrementado a un promedio de 82,8 (rango 40-95). Asimismo, el KSSf preoperatorio pasó de 48 (rango 25-60) a 85 (rango 55-100) en promedio (Tabla 2). El resultado fue "bueno-excelente" (>80) en 16 pacientes (64\%), "aceptable" en seis casos $(24 \%)$ y "pobre" en los tres restantes $(12 \%)$.

Respecto al RM, se incrementó de un promedio preoperatorio de 7,5 (rango 0-15)-76 (rango 10-110) a $1^{\circ}$ (rango 0-5)-102,4 (rango 85-115) en el último control. Tres pacientes tuvieron contractura en flexión $<5^{\circ}$ y uno, un déficit de extensión de $5^{\circ}$, ninguno requirió movilización bajo anestesia.

Se restauró el eje anatómico femorotibial (de $4^{\circ}$ a $7^{\circ}$ ) en todos los casos.

En el análisis radiográfico, se observó la adecuada posición de los componentes protésicos en todos los pacientes (Figura). Se detectaron tres rodillas con evidencia de demarcación tibial, con un seguimiento de 13.2 y 14.5 años, las cuales no eran progresivas durante los controles, y los pacientes no tenían síntomas hasta el final del seguimiento.

No se hallaron casos de necrosis cutánea/defectos tegumentarios, lesiones del aparato extensor, fractura o necrosis rotuliana, mal encarrilado femoro-rotuliano, inestabilidad clínica, trombosis venosa profunda/tromboembolismo pulmonar, lesiones neurovasculares ni distrofia simpática refleja en el posoperatorio.

Cuatro fueron operados nuevamente luego del RTR, con dos cirugías de revisión. Uno sufrió una infección superficial de la herida quirúrgica en el control de las tres semanas. Se le realizó una limpieza superficial y se le administró antibiótico, con una evolución favorable. Otro tuvo una infección hematógena aguda a los tres años del RTR, luego de un absceso en el tercio proximal del muslo homolateral; se realizó limpieza artroscópica más tratamiento antibiótico, con una buena evolución hasta el último control. Un paciente sufrió una fractura periprotésica femoral a los dos años del RTR; a pesar de la lazada de alambre profiláctica en el fémur distal (seudoartrosis de fractura de cóndilo femoral externo), se fracturó tras una caída de propia altura; se efectuó una revisión del RTR con colocación de tallo femoral cementado de mayor longitud que el anterior. 
Tabla 2. Datos demográficos de la serie

\begin{tabular}{|c|c|c|c|c|c|c|}
\hline Paciente & Sexo & Edad & Fractura & $\begin{array}{c}\text { KSS } \\
\text { preoperatorio }\end{array}$ & $\begin{array}{c}\text { KSS } \\
\text { posoperatorio }\end{array}$ & Complicaciones \\
\hline 1 & M & 74 & $\begin{array}{l}\text { Platillo tibial externo } \\
\text { izquierdo }\end{array}$ & 40 & 90 & \\
\hline 2 & $\mathrm{~F}$ & 87 & Platillo tibial externo derecho & 15 & 75 & \\
\hline 3 & $\mathrm{~F}$ & 82 & Supraintercondílea derecha & 25 & 80 & \\
\hline 4 & M & 78 & $\begin{array}{l}\text { Platillo tibial interno } \\
\text { izquierdo }\end{array}$ & 30 & 95 & \\
\hline 5 & $\mathrm{~F}$ & 69 & $\begin{array}{l}\text { Platillo tibial externo } \\
\text { izquierdo }\end{array}$ & 35 & 80 & \\
\hline 6 & M & 67 & $\begin{array}{l}\text { Platillo tibial externo } \\
\text { izquierdo }\end{array}$ & 35 & 80 & $\begin{array}{l}\text { Limpieza superficial + } \\
\text { antibióticos }\end{array}$ \\
\hline 7 & M & 38 & $\begin{array}{l}\text { Platillo tibial } \\
\text { externo izquierdo }+ \\
\text { supraintercondílea izquierda }\end{array}$ & 40 & 90 & \\
\hline 8 & $\mathrm{~F}$ & 69 & $\begin{array}{l}\text { Ambos platillos tibiales } \\
\text { izquierdos }\end{array}$ & 40 & 90 & \\
\hline 9 & M & 28 & $\begin{array}{l}\text { Platillo tibial interno } \\
\text { izquierdo (expuesta) }\end{array}$ & 50 & 80 & $\begin{array}{l}\text { Infección, limpieza } \\
\text { artroscópica }\end{array}$ \\
\hline 10 & M & 26 & Supraintercondílea izquierda & 50 & 95 & \\
\hline 11 & $\mathrm{~F}$ & 76 & $\begin{array}{l}\text { Cóndilo femoral } \\
\text { externo derecho }\end{array}$ & 55 & 80 & \\
\hline 12 & $\mathrm{~F}$ & 65 & Supraintercondílea derecha & 30 & 40 & $\begin{array}{l}\text { Fractura periprotésica, } \\
\text { revisión con tallo }\end{array}$ \\
\hline 13 & $\mathrm{~F}$ & 71 & $\begin{array}{l}\text { Platillo tibial externo } \\
\text { izquierdo }\end{array}$ & 45 & 95 & \\
\hline 14 & $\mathrm{~F}$ & 80 & $\begin{array}{l}\text { Ambos platillos tibiales } \\
\text { derechos }\end{array}$ & 30 & 55 & \\
\hline 15 & M & 75 & Platillo tibial interno derecho & 35 & 85 & \\
\hline 16 & $\mathrm{~F}$ & 65 & $\begin{array}{l}\text { Platillo tibial interno } \\
\text { izquierdo derecho }\end{array}$ & 35 & 85 & $\begin{array}{l}\text { Infección, espaciador en dos } \\
\text { tiempos }\end{array}$ \\
\hline 17 & $\mathrm{~F}$ & 48 & Platillo tibial externo derecho & 40 & 80 & \\
\hline 18 & $\mathrm{~F}$ & 49 & Supraintercondílea derecha & 45 & 95 & \\
\hline 19 & $\mathrm{~F}$ & 63 & Supraintercondílea izquierda & 45 & 90 & \\
\hline 20 & $\mathrm{~F}$ & 71 & Ambos platillos derechos & 40 & 90 & \\
\hline 21 & $\mathrm{~F}$ & 71 & Supraintercondílea derecha & 40 & 80 & \\
\hline 22 & $\mathrm{~F}$ & 67 & $\begin{array}{l}\text { Platillo tibial externo } \\
\text { izquierdo }\end{array}$ & 45 & 90 & \\
\hline 23 & $\mathrm{~F}$ & 85 & Supraintercondílea izquierda & 35 & 80 & \\
\hline 24 & $\mathrm{~F}$ & 38 & Ambos platillos derechos & 45 & 85 & \\
\hline 25 & $\mathrm{~F}$ & 69 & Supraintercondílea derecha & 40 & 85 & \\
\hline
\end{tabular}



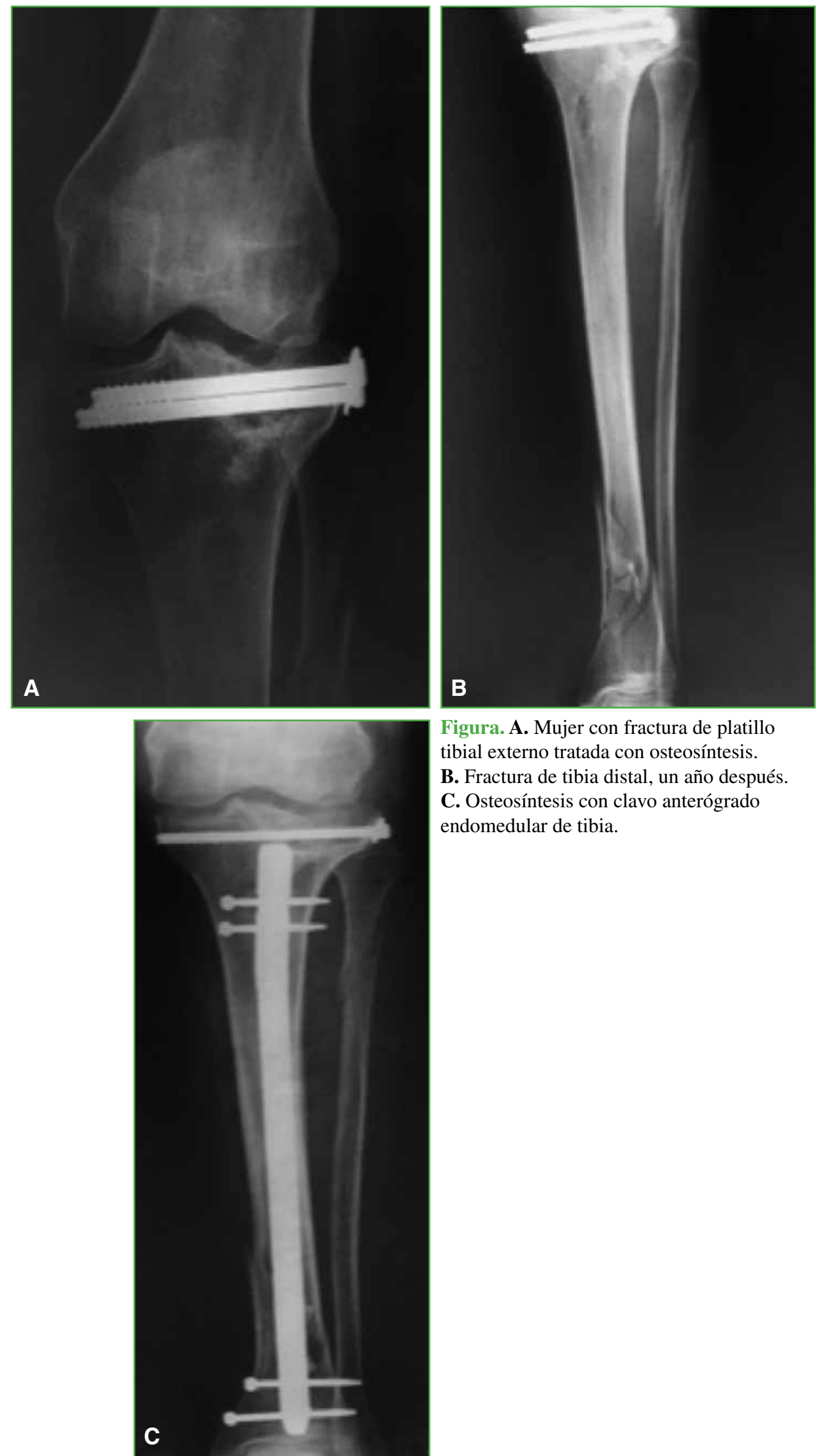

Figura. A. Mujer con fractura de platillo tibial externo tratada con osteosíntesis.

B. Fractura de tibia distal, un año después.

C. Osteosíntesis con clavo anterógrado endomedular de tibia. 

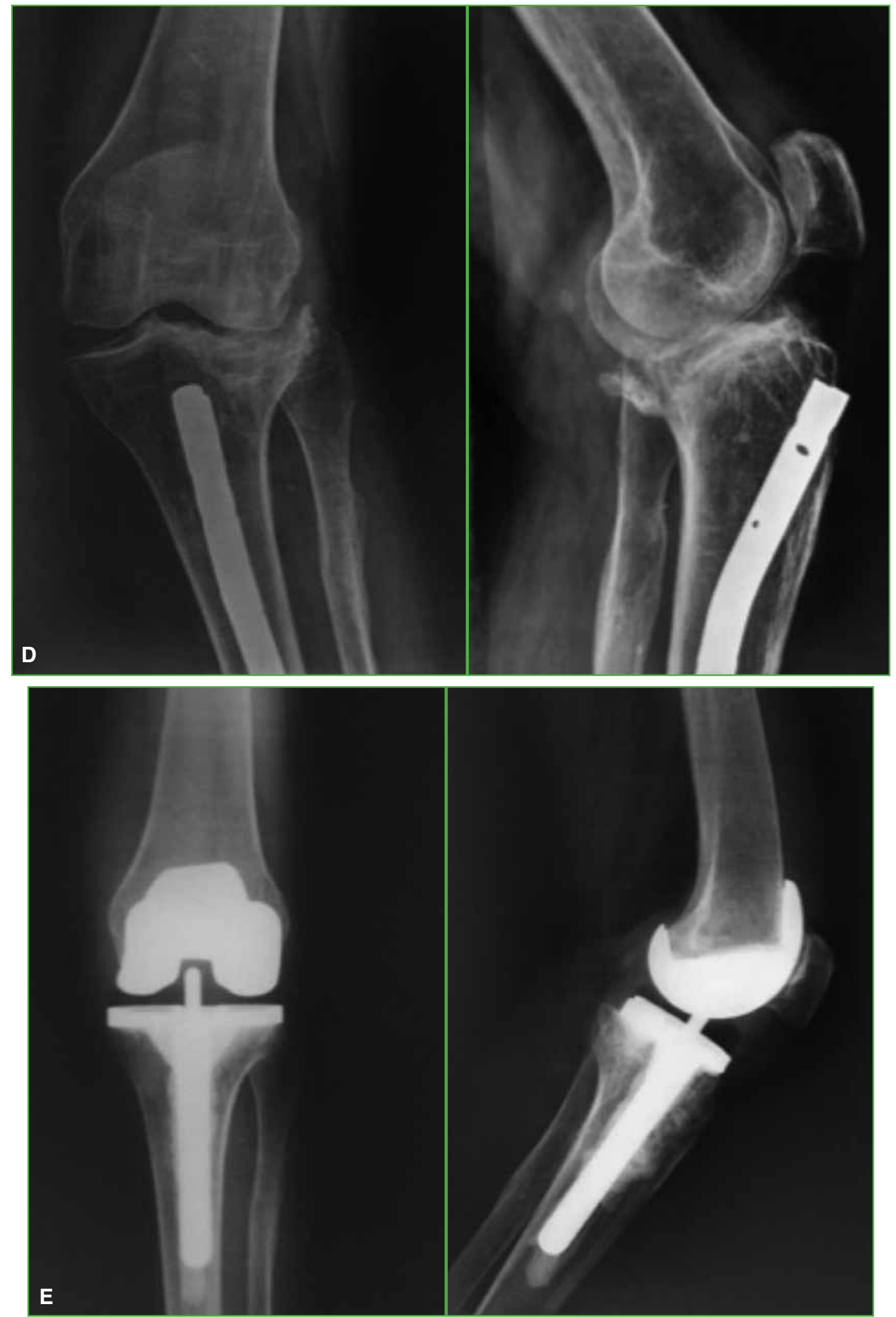

Figura. D. Retiro de tornillos canulados y toma de muestra, a los 4 meses. E. Artroplastia total de rodilla izquierda, con vástago tibial. 


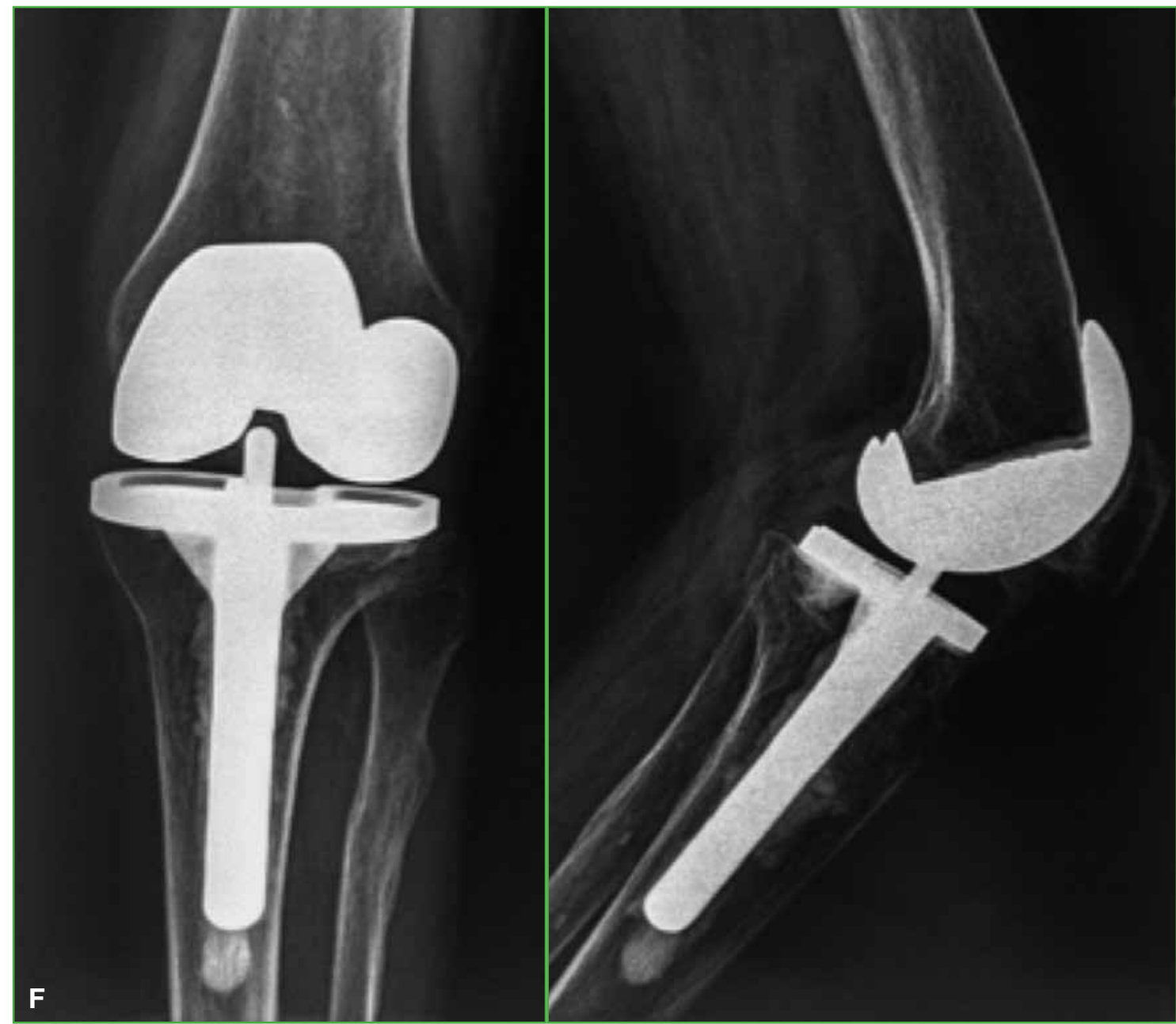

Figura. F. Evolución a los 7 años, sin signos de aflojamiento.

Por último, el paciente al que se le habían efectuado numerosas limpiezas por una infección del sitio quirúrgico de su fractura de platillo tibial interno, tratamiento de seudoartrosis y cirugía de cobertura de partes blandas, sufrió una infección subaguda de la prótesis (cultivos preoperatorios negativos), con cultivo positivo de Staphylococcus resistente a meticilina. Fue sometido a una revisión en dos tiempos con colocación de espaciador prefabricado a los 35 días del RTR y conversión a nueva prótesis a los 4.5 meses. El paciente concurrió al control final con análisis de laboratorio y punción negativos para infección, pero manifestó dolor y limitación funcional, a los 5 años y 3 meses de la revisión de rodilla. Se sospecha que persiste la infección (paciente con antecedentes de dependencia de drogas endovenosas).

De acuerdo con este análisis, la supervivencia de la prótesis fue del 92\% a los 6.1 años (rango 4.3-16.2) de seguimiento promedio, considerando la necesidad de revisión por cualquier causa. Si discriminamos la causa mecánica, asciende al $96 \%$.

\section{DISCUSIÓN}

Sobre 25 RTR en pacientes con secuela de fracturas articulares, se obtuvo un incremento considerable en lo que respecta al KSS y al RM, con restauración del eje anatómico en todos los casos, una tasa de reoperación del 16\% y una supervivencia de la prótesis del $92 \%$ a los 6.1 años de seguimiento promedio.

Los resultados del RTR en pacientes con gonartrosis como secuela de fractura articular son inferiores a aquellos que se obtienen en artroplastias primarias convencionales por artrosis idiopática (90-95\% a 10-15 años), con publicaciones que avalan dicha afirmación. ${ }^{11,12}$ 
Existe escasa información sobre la supervivencia de la prótesis a mediano o largo plazo. Entre las primeras publicaciones sobre el tema, se destacan los trabajos de Roffi y Merritt, quienes obtuvieron pobres resultados interviniendo pacientes con secuela de fractura articular de rodilla, con un seguimiento promedio de 27 meses (rango 1-4 años) y un 38,5\% de malos resultados. Asimismo, Lonner y cols. observaron una tasa más alta de complicaciones en pacientes tratados con RTR por gonartrosis postraumática y comunicaron buenos resultados solo en el $71 \%$ de los casos. ${ }^{9}$

En publicaciones más actuales, Saleh y cols., en una evaluación retrospectiva, obtuvieron un $80 \%$ de resultados buenos-excelentes en 15 RTR operados con un mínimo de cinco años de seguimiento. Sin embargo, tres pacientes $(20 \%)$ desarrollaron una infección profunda, por lo que dos fueron sometidos a artrodesis y el restante, a cirugía de revisión en dos tiempos, con una supervivencia de la prótesis del $80 \%$ a los 6.2 años de seguimiento promedio. ${ }^{4}$ Un estudio prospectivo de Weiss y cols. analizó 62 RTR por gonartrosis después de una fractura de platillos tibiales, con una tasa de reoperación del $21 \%$ (5 revisiones por causa infecciosa y mecánica); la supervivencia fue del $91,9 \%$ a los 4.7 años de seguimiento promedio. ${ }^{5}$ De manera similar, en otro estudio multicéntrico de Salcedo Zunino y cols., ${ }^{13}$ se analizaron, en forma retrospectiva, 29 rodillas sometidas a RTR por artrosis como secuela de un trauma articular, entre 2008 y 2015, con un seguimiento promedio de 45 meses. La tasa de complicaciones fue del $21 \%$ y la de supervivencia de la prótesis, del $93,1 \%$. Recientemente, Lizaur-Utrilla y cols. ${ }^{14}$ publicaron un estudio de cohorte prospectivo, en el que compararon pacientes operados con diagnóstico de artrosis idiopática con aquellos intervenidos por gonartrosis postraumática. Si bien no hallaron diferencias estadísticamente significativas en cuanto a la función y la movilidad, el grupo operado por artrosis postraumática tuvo una tasa más alta de complicaciones.

Nuestra serie, que coincide con las publicaciones actuales, muestra resultados similares, con una supervivencia apropiada a mediano plazo, a pesar de la tasa de reoperación elevada (Tabla 3).

Las probables razones por las cuales estos casos conducen a resultados menos favorables, con una tasa más alta de complicaciones luego del RTR incluyen: artroplastia de rodilla en pacientes jóvenes con mayor demanda funcional (artrosis prematura por la extensión articular de la fractura), osteosíntesis previa, seudoartrosis, probabilidad de infección latente, defectos óseos intrarticulares y mala alineación residual, tiempo quirúrgico más prolongado, cirugías previas al RTR que pueden comprometer la cobertura de partes blandas y predisponer a complicaciones cutáneas, y fibrosis cicatricial intrarticular relacionada con el trauma inicial que puede complicar la exposición quirúrgica, el balance ligamentario y afectar el RM. ${ }^{8,15,16}$

Tabla 3. Comparación de las distintas variables

\begin{tabular}{|c|c|c|c|c|c|}
\hline Series & Seguimiento & $\begin{array}{c}\text { Tasa de } \\
\text { complicaciones }\end{array}$ & $\begin{array}{l}\text { Supervivencia } \\
\text { de la prótesis }\end{array}$ & $\begin{array}{c}\text { Rango de movilidad } \\
\text { preoperatorio }\end{array}$ & $\begin{array}{c}\text { Rango de movilidad } \\
\text { posoperatorio }\end{array}$ \\
\hline $\begin{array}{l}\text { Roffi y cols. } \\
(1990)^{23}\end{array}$ & $\begin{array}{c}27 \text { meses } \\
(12-48)\end{array}$ & $38,4 \%$ & - & - & \\
\hline Lonner $^{9}$ (1999) & $\begin{array}{l}46 \text { meses } \\
(28-114)\end{array}$ & $57,0 \%$ & $71 \%$ & $\begin{array}{c}94^{\circ} \\
\left(20-120^{\circ}\right)\end{array}$ & $\begin{array}{c}100^{\circ} \\
\left(0-135^{\circ}\right)\end{array}$ \\
\hline $\begin{array}{l}\text { Saleh y cols. }{ }^{4} \\
(2001)\end{array}$ & $\begin{array}{l}6.2 \text { años } \\
(5.4-11.1)\end{array}$ & $53,3 \%$ & $80 \%$ & $\begin{array}{c}87^{\circ} \\
\left(20-125^{\circ}\right)\end{array}$ & $\begin{array}{c}105^{\circ} \\
\left(70-135^{\circ}\right)\end{array}$ \\
\hline $\begin{array}{l}\text { Weiss y cols. } \\
(2003)\end{array}$ & 4.7 años & $36 \%$ & $79 \%$ & - & - \\
\hline $\begin{array}{l}\text { Salcedo Zunino } \\
\text { y cols. }{ }^{13}(2015)\end{array}$ & $\begin{array}{l}45 \text { meses } \\
(6-104)\end{array}$ & $21 \%$ & $93,1 \%$ & $\begin{array}{c}60^{\circ} \\
\left(45-90^{\circ}\right)\end{array}$ & $\begin{array}{c}112 \\
\left(60-140^{\circ}\right)\end{array}$ \\
\hline $\begin{array}{l}\text { Nuestra serie } \\
(2017)\end{array}$ & $\begin{array}{l}6.1 \text { años } \\
(4.3-16.2)\end{array}$ & $16 \%$ & $92 \%$ & $\begin{array}{c}76^{\circ} \\
\left(10-110^{\circ}\right)\end{array}$ & $\begin{array}{c}102,4^{\circ} \\
\left(85-115^{\circ}\right)\end{array}$ \\
\hline
\end{tabular}


Si bien el RTR, en estos casos, es técnicamente demandante, destacamos la importancia de obtener una adecuada alineación posoperatoria y conseguir un posicionamiento correcto de los componentes protésicos, en íntima relación con el logro de un buen resultado posquirúrgico; hay estudios que aseveran este concepto. ${ }^{17,18}$ Se demostró que la inapropiada colocación de componentes tiene implicancias negativas en la supervivencia de la prótesis a largo plazo. En el estudio de Weiss y cols., ${ }^{5}$ los casos con posicionamiento subóptimo de los componentes o deseje residual se asociaron con pobres resultados, comparados con las rodillas con aceptable restauración de la alineación. En nuestra evaluación, la alineación y la colocación de los componentes protésicos fueron adecuadas en todos los pacientes.

La tasa de infección en estos pacientes es más alta al compararla con lo descrito por diferentes autores respecto de RTR intervenidos por gonartrosis idiopática, con una prevalencia que oscila entre el $1 \%$ y el $2 \%$ (Tabla 4 ). ${ }^{19-21}$

Tabla 4. Series detalladas en orden cronológico, con sus casos y tasas de infección respectivos

\begin{tabular}{|l|c|c|}
\hline Autor & Cantidad de pacientes & Tasa de infección \\
\hline Lonner (1999) & 25 & $10 \%$ \\
\hline Saleh y cols. (2001) & 15 & $15 \%$ \\
\hline Weiss y cols. (2003) & 26 & $3,2 \%$ \\
\hline Civinini y cols. (2009) & 30 & $4 \%$ \\
\hline Massin (2011) & 40 & $5 \%$ \\
\hline Nuestra serie & 25 & $4 \%$ \\
\hline
\end{tabular}

Al igual que los estudios de Weiss y cols. ${ }^{11}(3,2 \%)$ y Civinini y cols. ${ }^{22}$ (4\%), obtuvimos una tasa relativamente baja de infección de la prótesis (4\%), teniendo en cuenta que otros dos casos fueron de origen hematógeno agudo y superficial (este último sin comprometer al implante), en comparación con las series de Lonner y cols. ${ }^{9}$ y de Saleh y cols., ${ }^{4}$ con una tasa del $10 \%$ y 15\%, respectivamente. Una explicación probable para entender esta tasa más baja de infección es que nuestra serie incluyó fracturas articulares de rodilla que recibieron tratamiento incruento.

Nuestra práctica diaria incluye un algoritmo de estudios preoperatorios con el objetivo de descartar una infección oculta en este tipo de cuadro. Luego de un minucioso examen físico, se procede a los análisis de sangre de rutina (recuento de glóbulos blancos, eritrosedimentación y proteína $\mathrm{C}$ reactiva cuantitativa). Ante la sospecha de infección, se realiza la punción/aspiración de líquido articular y se toman muestras de tejido óseo (este procedimiento se efectúa cuando se decide realizar el RTR en dos tiempos, con previa extracción de la osteosíntesis). A pesar de los recaudos detallados, el único caso de infección profunda (paciente con múltiples operaciones y diagnóstico de infección, seudoartrosis y defecto de cobertura) que requirió una cirugía de revisión en dos tiempos, arrojó resultados negativos para infección en la punción y la muestra ósea antes del RTR.

Una complicación frecuente es el retraso de la cicatrización y la infección de la herida quirúrgica. De manera similar a Weiss y cols., ${ }^{5}$ con tres limpiezas más terapia antibiótica por infección superficial $(4,8 \%)$, uno de nuestros pacientes desarrolló la complicación a las tres semanas del RTR, con idéntica resolución (4\%).

Debido a las distintas variables en juego, esta cirugía plantea una demanda técnica elevada. Por ello, la planificación preoperatoria constituye un instrumento imprescindible al abordar estos casos. Durante el examen físico, se deben valorar el estado tegumentario (incisiones previas), el estado neurovascular, el RM y el grado de estabilidad de la rodilla. Se debe elegir cuidadosamente y a conciencia el sitio del abordaje cutáneo, manteniendo un puente de 5-7 cm en relación con las cicatrices previas. La evaluación preoperatoria de la rótula puede dar una idea de la dificultad para el abordaje; una rótula fija en extensión, con una flexión limitada $<90^{\circ}$, indica una alta probabilidad de realizar algún gesto sobre el aparato extensor para una adecuada exposición. Las radiografías convencionales muestran la osteosíntesis previa, la alineación residual y la presencia de defectos óseos, los que pueden estar disimulados por el material de osteosíntesis previo, por lo cual puede ser útil recurrir a una tomografía axial computarizada en dicha planificación. En los casos de inestabilidad clínica, se requieren radiografías con estrés varovalgo, a fin de determinar el grado de estabilidad articular y elegir el grado de constreñimiento del implante por 
colocar. En esta etapa de planificación, es preciso determinar, además, la necesidad de suplementación (cemento, hueso o suplementos metálicos) y el uso de tallos femoral o tibial. Es recomendable utilizar tallos que salteen el defecto del tornillo tibial más distal o femoral más proximal en aquellos pacientes en los que se retire el material de osteosíntesis.

Las debilidades de este estudio son su diseño retrospectivo, el período prolongado de recolección de datos, el escaso número de pacientes de la muestra y los diferentes tipos de fracturas con tratamientos dispares. La fortaleza es el tiempo de seguimiento posoperatorio.

\section{CONCLUSIONES}

El RTR es un tratamiento adecuado para la gonartrosis postraumática en su estadio final, con buenos resultados a mediano plazo. Sin embargo, el ortopedista debe estar al tanto de la tasa más alta de complicaciones, en comparación con la artroplastia primaria por gonartrosis idiopática. Dada la complejidad de estos casos, se recomienda realizar una detallada planificación preoperatoria. Algunos pacientes requerirán prótesis con mayor grado de constreñimiento, suplementos metálicos y tallos femorales/tibiales, implantes en ocasiones reservados para revisiones de rodilla complejas. Si se logran una adecuada alineación posoperatoria y un correcto posicionamiento de los componentes protésicos, los resultados son satisfactorios y se asemejan a los del RTR de rutina. Queda pendiente la evaluación de los resultados a más largo plazo, considerando que se trata de pacientes jóvenes, con elevada demanda funcional y expectativa de vida, en quienes se han utilizado implantes de revisión en algunos casos, lo cual sugiere posibles revisiones a futuro.

Conflicto de intereses: Los autores no declaran conflictos de intereses.

ORCID de L. Pérez Alamino: http://orcid.org/0000-0002-1563-6947

ORCID de S. Aguer: http://orcid.org/0000-0001-9079-1966

ORCID de G. Garabano: http://orcid.org/0000-0001-5936-0607

ORCID de H. del Sel: http://orcid.org/0000-0002-3655-1408

ORCID de F. Lopreite: http://orcid.org/0000-0002-2065-8649

\section{BIBLIOGRAFÍA}

1. Saleh H, Yu S, Vigdorchik J, Schwarzkopf R, Saleh H, Yu S, et al. Total knee arthroplasty for treatment of posttraumatic arthritis: Systematic review. World J Orthop 2016;7(9):584-91. https://doi.org/10.5312/wjo.v7.i9.584

2. Massin P. Total knee replacement in post-traumatic arthritic knees with limitation of flexion. Orthop Traumatol Surg Res [Internet]. 2011;97(1):28-33. https://doi.org/10.1016/j.otsr.2010.06.016

3. Putman S, Argenson J, Bonnevialle P, Ehlinger M, Vie P, Leclercq S, et al. Ten-year survival and complications of total knee arthroplasty for osteoarthritis secondary to trauma or surgery: A French multicentre study of 263 patients. Orthop Traumatol Surg Res 2018;104:161-4. https://doi.org/10.1016/j.otsr.2017.11.019

4. Saleh K, Sherman P, Katkin P, Windsor R, Haas S, Laskin R, Sculco P. Total knee arthroplasty after open reduction and internal fixation of fractures of the tibial plateau. J Bone Joint Surg (Am) 2001;83(8):1144-8. https://doi.org/10.2106/00004623-200108000-00002

5. Weiss NG, Parvizi J, Hanssen AD, Trousdale RT, Lewallen DG. Total knee arthroplasty in post-traumatic arthrosis of the knee. J Arthroplasty 2003;18(3):23-6. https://doi.org/10.1054/arth.2003.50068

6. El-galaly A, Haldrup S, Pedersen AB, Kappel A, Jensen MU, Nielsen PT. Increased risk of early and medium-term revision after post-fracture total knee arthroplasty. Results from the Danish Knee Arthroplasty Register. Acta Orthop 2017;88(3):263-8. https://doi.org/10.1080/17453674.2017.1290479

7. Papadopoulos EC, Parvizi J, Lai CH, Lewallen DG. Total knee arthroplasty following prior distal femoral fracture. Knee 2002;9:267-74. https://doi.org/10.1016/s0968-0160(02)00046-7

8. Elsoe R, Johansen MB, Larsen P. Tibial plateau fractures are associated with a long-lasting increased risk of total knee arthroplasty a matched cohort study of 7,950 tibial plateau fractures. Osteoarthr Cartil [Internet] 2018. https://doi.org/10.1016/j.joca.2018.12.020 
9. Lonner JH, Pedlow FX, Siliski JM. Total knee arthroplasty for post-traumatic osteoarthritis. J Arthroplasty 1999;14(8):969-75. https://doi.org/10.1016/s0883-5403(99)90012-8

10. Coonse KD, Adams JD. A new operative approach for the knee joint. Surg Gynecol Obs 1943;77:344-7.

11. Weiss NG, Parvizi J, Trousdale RT, Bryce RD, Lewallen DG. Total knee arthroplasty in patients with a prior fracture of the tibial plateau. J Bone Joint Surg 2003;85(2):218-21. https://doi.org/10.2106/00004623-200302000-00006

12. Meftah M, Jhurani A, Bhat JA, Ranawat AS, Ranawat CS. The effect of patellar replacement technique on patellofemoral complications and anterior knee pain. J Arthroplasty [Internet] 2012;27(6):1075-1080.e1. http://dx.doi.org/10.1016/j.arth.2011.10.006

13. Salcedo Zunino LA, Gutierrez Olivera N, Pioli I, Bidolegui F, Vindver G, Allende BL. Reemplazo total de rodilla en secuela de fracturas alrededor de la rodilla. Rev Asoc Arg Ortop Traumatol 2017;82(2):102-8. https://doi.org/10.15417/551

14. Lizaur-Utrilla A, Collados-Maestre I, Miralles-Muñoz FA, Lopez-Prats FA. Total knee arthroplasty for osteoarthritis secondary to fracture of the tibial plateau . A prospective matched cohort study. J Arthroplasty 2015;30(8):1329-32. https://doi.org/10.1016/j.arth.2015.02.032

15. Manidakis N, Dosani A, Dimitriou R, Stengel D, Matthews S, Giannoudis P. Tibial plateau fractures: functional outcome and incidence of osteoarthritis in 125 cases. Int Orthop 2010;34(4):565-70. https://doi.org/10.1007/s00264-009-0790-5

16. Gehrke T, Parvizi J. Proceedings of the International Consensus Meeting on Periprosthetic Joint Infection. https://www.efort.org/wp-content/uploads/2013/10/Philadelphia_Consensus.pdf

17. Khoshbin A, Stavrakis A, Sharma A, Woo P. Patient-reported outcome measures of total knee arthroplasties for post-traumatic arthritis versus osteoarthritis: A short-term (5- to 10-year) retrospective matched cohort study. J Arthroplasty 2019;34(5):P872-6. https://doi.org/10.1016/j.arth.2019.01.022

18. Lunebourg A, Parratte S, Gay A, Ollivier M, Garcia-Parra K, Argenson JN. Lower function, quality of life, and survival rate after total knee arthroplasty for posttraumatic arthritis than for primary arthritis. Acta Orthop 2015;86(2):189-94. https://doi.org/10.3109/17453674.2014.979723

19. Gaine WJ, Ramamohan NA, Hussein NA, Hullin MG, McCreath SW. Wound infection in hip and knee arthroplasty. J Bone Joint Surg Br 2000;82(4):561-5. https://doi.org/10.1302/0301-620x.82b4.10305

20. Chun KC, Kim KM, Chun CH. Infection following total knee arthroplasty. Knee Surg Relat Res 2013;25(3):93-9. https://doi.org/10.5792/ksrr.2013.25.3.93

21. Suzuki G, Saito S, Ishii T, Motojima S, Tokuhashi Y, Ryu J. Previous fracture surgery is a major risk factor of infection after total knee arthroplasty. Knee Surg Sports Traumatol Arthrosc 2011;19(12):2040-4. https://doi.org/10.1007/s00167-011-1525-x

22. Civinini R, Carulli C, Matassi F, Villano M, Innocenti ML. Total knee arthroplasty after complex tibial plateau fractures. Chir Organi Mov 2009;93(3):143-7. https://doi.org/10.1007/s12306-009-0033-3 